\title{
¿Por qué México tiene que orientar su crecimiento económico hacia a las exportaciones en el T-MEC? evidencia empírica 1993-2019
}

\author{
Héctor F. Salazar-Núñez $\quad$ José Antonio Lozano-Díez** \\ Francisco Venegas-Martínez ${ }^{* * *}$
}

(Recibido: noviembre, 2019/Aceptado: marzo, 2020)

\section{Resumen}

Esta investigación examina la existencia de un vínculo causal entre el Producto Interno Bruto y Exportaciones para los países que integran el T-MEC con base en técnicas de cointegración y causalidad de Granger. El análisis se extiende al agregar el método de estimación de mínimos cuadrados ordinarios totalmente modificados (FMOLS). La evidencia empírica muestra que el Producto Interno Bruto y Exportaciones de Canadá, México y EU. son I(1), pero al aplicar pruebas de cointegración, sólo las variables pertenecientes a México estaban cointegradas. Por último se aplican pruebas de causalidad y se encuentra que el Producto Interno Bruto y las Exportaciones de México tienen una relación causal bidireccional. Por lo tanto, las políticas económicas destinadas a favorecer las exportaciones, también favorecerán al crecimiento económico y viceversa.

Palabras clave: exportaciones, crecimiento económico; modelos series de tiempo; cointegración; métodos econométricos.

Clasificación JEL: F1, O4, C22, C32, C18.

\footnotetext{
*Profesor-investigador en la Universidad Cristóbal Colón, México. <hectorsalazar313@gmail.com>.

** Profesor-investigador en la Universidad Panamericana, México. <jlozano@up.edu.mx>.

*** Profesor-investigador en el Instituto Politécnico Nacional, México. <fvenegas1111@yahoo.com.mx>
} 


\title{
Why does Mexico have to direct its economic growth towards exports in the T-MEC? empirical evidence 1993-2019
}

\begin{abstract}
This paper is aimed at examining if there is a causal link between Gross Domestic Product and Exports for the countries that integrate the T-MEC on the basis of cointegration and Granger-causality techniques. The analysis is also extended by using the estimation method of fully modified ordinary least squares (FMOLS). Empirical evidence shows that Gross Domestic Product and Exports of Canada, Mexico and USA are I(1), but when applying the cointegration tests, only the variables belonging to Mexico were cointegrated. Finally, the causality test is applied and it was found that Gross Domestic Product and Exports of Mexico have a causal relationship of a bidirectional type. Therefore, economic policies destined to favor exports will also favor economic growth and vice versa.
\end{abstract}

Keywords: exports, economic growth; time series models; cointegration; econometric methods.

JEL classifications: $\mathrm{F} 1, \mathrm{O} 4, \mathrm{C} 22, \mathrm{C} 32, \mathrm{C} 18$.

\section{Introducción}

La presente investigación realiza el análisis del efecto del crecimiento de las exportaciones, ${ }^{1}$ sobre el crecimiento económico en Canadá (CA), México (MX) y Estados Unidos de Norteamérica (US). Las primeras investigaciones de este tipo fueron realizadas por Michaely (1977) y Balassa (1978). El primero prueba la hipótesis, "un rápido crecimiento en las Exportaciones, genera un acelerado crecimiento económico", mientras el segundo se concentra en la hipótesis "las políticas orientadas a la exportación generan un mayor crecimiento económico que las políticas que favorecen la sustitución

\footnotetext{
${ }^{1}$ Aunque se diga Exportaciones en realidad serán solo las exportaciones de bienes, a menos que se diga algo diferente.
} 
de importaciones". En ambos casos se ajustan a la hipótesis de crecimiento orientado hacia las Exportaciones que ha tenido un auge durante las últimas décadas en México y el mundo, dado que también han sido políticas sugeridas por organizamos internacionales para promover el crecimiento económico, tales como el Banco mundial (1987). Por otro lado, existen otras vertientes sobre el mismo tema, en donde le dan una mayor importancia a la inversión extranjera directa y al turismo como motor del crecimiento económico; por ejemplo véase a Borensztein (1998) y Shan y Wilson (2001).

La investigación realizada por Michaely (1977) en el estudio de este fenómeno económico analiza 41 países donde utilizo el coeficiente de correlación para probar la relación entre el crecimiento de las Exportaciones y económico, encontrando que en siete países (Grecia, Taiwán, Portugal, España, Israel, Yugoslavia y Corea), el coeficiente de correlación era altamente significativo para el periodo de estudio 1950-1973. Mientras que Balassa (1978) realizó la misma tarea que Michaely (1977), sólo que trató de homogeneizar a los países que introdujo en el estudio de ahí que encontró una mayor correlación entre las variaciones de las Exportaciones y el crecimiento económico durante 19601973, aunque de acuerdo a Tyler (1981), el estudio sólo consideró a once países. Por último, Rodríguez-Benavides y Venegas-Martínez (2011) estudian los efectos de las exportaciones en el crecimiento económico de México a través un análisis de cointegración en el periodo 1929-2009.

Los métodos econométricos se renovaron a finales de la década de los ochenta con la entrada de la metodología de cointegración, propuesta por Engle y Granger (1987) para modelos uniecuacionales, seguida por la propuesta por Johansen $(1988,1991)$ aplicada a vectores autorregresivos; junto con las pruebas de causalidad en ambos casos. Aunado a esto se mejoraron los métodos de estimación al surgir los mínimos cuadrados ordinarios totalmente modificados (FMOLS, por sus siglas en inglés) propuesto por Phillips y Hansen (1990) y mínimos cuadrados ordinarios dinámicos (DOLS, por sus siglas en inglés) por Stock y Watson (1993), entre otros. Aunque esta investigación sólo abarcara el primer método, el cual se ocupa para corregir dos fenómenos: 1) la correlación entre la ecuación de cointegración y las innovaciones de las variables exógenas y 2) la endogeneidad de largo plazo, para lo cual se realiza una corrección semiparamétrica a la varianza de largo plazo.

Existen diversos estudios empíricos que han tratado de probar la hipótesis mencionada anteriormente que hacen uso de la metodología de cointegración, 
ya sea para modelos uniecuacionales o vectores autoregresivos. Entre los primeros trabajos de investigación que emplearon la metodología de cointegración se encuentra el realizado por Kwan y Cotsomitis (1991), lo aplican a China para el periodo 1952-1985 en donde encuentran una relación bidireccional entre las exportaciones y el ingreso nacional. Marin (1992) estudia a cuatro países industrializados, Alemania, Estados Unidos de Norteamérica Japón y Reino Unido, y concluye que la hipótesis no puede ser rechazada para estos países en el periodo 1967-1987 con datos periodicidad trimestral. Shan y Sun (1998: a) aplican la metodología de cointegración de Johansen a Australia de 1978-1993 con periodicidad trimestral, utilizando variables en forma de variaciones de la formación bruta de capital, empleo, exportaciones e importaciones y como variable endógena al producto manufacturero de exportación, rechazando la hipótesis para este país. Shan y Sun (1998, b) realizan un resumen de treinta trabajos realizados desde 1978 hasta 1996 de las diversas metodologías utilizadas y conclusiones a las que se han llegado, siendo el 75\% a favor de la hipótesis. Dhawan y Biswal (1999) realizan el estudio aplicándolo a la India, utilizando la metodología de Johansen a las variables: exportaciones, Producto Interno Bruto y términos de intercambio, y encuentran que existe una relación bidireccional entre las exportaciones y el producto en el corto y largo plazo para el periodo 1961-1993. Panas y Vamvoukas (2002) estudian la hipótesis aplicando la metodología de cointegración con el modelo de vectores autorregresivos a Grecia, añadiendo variables como el tipo de cambio e índice de precio al consumidor a la hipótesis, teniendo como conclusión el rechazo de esta para el periodo 1948-1997. Awokuse (2003), encuentra que la hipótesis se acepta para Canadá, utilizando el modelo de vectores cointegrados haciendo uso de la función Cobb-Douglas y aumentado las variables: términos de intercambio, exportaciones y producto extranjero, para el periodo 1961-2000 con datos trimestrales. Shirazi y Manap (2005), analizan cinco economías del sur de Asia, como son Paquistán, India, Bangladesh, Sri Lanka y Nepal para el periodo 1960-2003, encontrado que la hipótesis se cumple en cuatro países (excepción de Sri Lanka). Las últimas investigaciones publicadas han sido las de Awokuse (2008) que examina el impacto de las exportaciones e importaciones sobre el crecimiento económico de Perú, Argentina y Colombia, con diversos periodos de inicio del estudio y terminación en 2002, con la metodología de vectores autoregresivos cointegrados, encontrando que tanto las exportaciones como importaciones mantienen una relación 
de largo plazo, para lo cual utilizo una función de producción neoclásica. Como se observa, los diversos estudios realizados, la metodología de Engle y Granger o de Johansen, sin tomar en cuenta al método de estimación, por eso en este documento se implementará la metodología de cointegración Engle y Granger en 1987, junto con el método de estimación propuesto por Phillips y Hansen (1990).

Este trabajo estudia la hipótesis de crecimiento orientado hacia las exportaciones de México, utilizando la metodología de cointegración Engle y Granger (1987), junto con el método de estimación propuesto por Phillips y Hansen (1990). En la segunda sección, se presenta de manera breve el método de estimación. En la tercera sección se realiza un análisis estadístico, correlación, pruebas de raíces unitarias y de estacionariedad. En la cuarta sección se realiza la estimación del modelo por el método propuesto, y se aplican las pruebas de cointegración y de causalidad de Granger. Por último, se presentan las conclusiones.

\section{Estimación por mínimos cuadrados ordinarios totalmente modificados (FMOLS)}

El método FMOLS fue propuesto por Phillips y Hansen (1990), en dicho método se propone corregir dos fenómenos. Primero la correlación entre la ecuación de cointegración y las innovaciones de las variables exógenas y segundo la endogeneidad de largo plazo. Para esto se realiza una corrección semiparamétrica. En primer lugar se plantea una representación triangular estándar de una regresión y se supone la existencia de al menos un vector de cointegración.

$$
\begin{aligned}
& y_{t}=X_{t}^{\prime} \beta+\varepsilon_{1 t} \\
& X_{t}=Z_{t}^{\prime} \alpha+\mu_{2 t} \\
& \Delta \mu_{2 t}=\varepsilon_{2 t}
\end{aligned}
$$

donde el vector $y_{t}$ es la variable independiente, $X_{t}$ es la matriz que contiene las variables exógenas, la constante y la tendencia según sea el caso $\left(Z_{t}\right.$ se explica de forma similar), $\beta$ es el vector de parámetros asociados a la matriz $X_{t}, \alpha$ son los parámetros asociado a la matriz $Z_{t}$. El vector de innovaciones $\varepsilon_{t}=\left(\varepsilon_{1 t}, \varepsilon_{1 t}\right)^{\prime}$ es estacionario y ergódico con media cero $\mathrm{y}$ 
matriz de varianza-covarianza finita $\Sigma>0$, matriz de covarianzas de largo plazo $\Omega>0$ de dos lados y la matriz de covarianzas de largo plazo $\Lambda$ de un lado. Además, Phillips y Hansen (1990) suponen que la suma parcial con la que se construye $\varepsilon_{t}$ satisface el principio de invariancia multivariada propuesta por Phillips y Durlauf (1986).

Por otro lado, la matriz de covarianzas $\Omega$ de largo plazo (dos lados) se puede descomponer de la siguiente forma:

$$
\begin{aligned}
\Omega & =\left(\begin{array}{ll}
\Omega_{11} & \Omega_{12} \\
\Omega_{21} & \Omega_{22}
\end{array}\right)=\Sigma+\Lambda+\Lambda^{\prime} \\
\Delta & =\left(\begin{array}{ll}
\Delta_{11} & \Delta_{12} \\
\Delta_{21} & \Delta_{22}
\end{array}\right)=\Sigma+\Lambda \\
\Lambda & =\sum_{j=0}^{\infty} E\left(\varepsilon_{t} \varepsilon_{t-j}^{\prime}\right)=\left(\begin{array}{ll}
\Lambda_{11} & \Lambda_{12} \\
\Lambda_{21} & \Lambda_{22}
\end{array}\right) \\
\Sigma & =E\left(\varepsilon_{t} \varepsilon_{t}^{\prime}\right)=\left(\begin{array}{ll}
\Sigma_{11} & \Sigma_{12} \\
\Sigma_{21} & \Sigma_{22}
\end{array}\right)
\end{aligned}
$$

aunque existen diversos métodos para calcular las matrices de covarianzas de largo plazo $\Omega$ y $\Lambda$, en este trabajo se utilizarán métodos no paramétricos tal como los propuestos por Newey y West (1987) y Andrews (1991). ${ }^{2}$

Los métodos no paramétricos utilizan Kernels y métodos para calcular el ancho de banda para estimar la covarianza de largo plazo. ${ }^{3}$ No obstante, en este trabajo se utilizarán los Kernels tipo Bartlett y el ancho de banda será fijo como lo proponen Newey y West (1987), el cual depende del tamaño de la muestra.

Para estimar el modelo dado en (1) por medio del método FMOLS, se utilizará a las variables Producto Interno Bruto $\left(Y_{t}\right)$ y las Exportaciones $\left(X_{t}\right)$ de los países, denominada en moneda corriente, para un periodo 1993-2019, obtenidas de la Organización para la Cooperación y Desarrollo Económico (OECD). El estimador del modelo FMOLS está definido por:

\footnotetext{
${ }^{2}$ Se pueden consultar Haan y Levin (1997) para métodos paramétricos y Andrews and Monahan (1992) y Lee y Phillips (1994) para métodos híbridos.

${ }^{3}$ Wang y Hu (2012) se puede encontrar un resumen de las principales características de las funciones de Kernels y el ancho de banda que se pueden utilizar
} 


$$
\hat{\theta}=\hat{\beta}=\left(\sum_{t=1}^{T} x_{t} x_{t}^{\prime}\right)^{-1}\left(\sum_{t=1}^{T} x_{t} y_{t}^{*}-T \hat{\Lambda}_{12}^{*}{ }^{\prime}\right)
$$

donde $x_{t}=\left(X_{t}, Z_{t}^{\prime}\right)^{\prime}$ de acuerdo con la ecuación 2. El sesgo de la variable $y_{t}$ es corregido por $\Omega_{12} \Omega_{22}^{-1} \varepsilon_{2 t}$ de manera que $y_{t}^{*}$ se define:

$$
y_{t}^{*}=y_{t}-\widehat{\Omega}_{12} \hat{\Omega}_{22}^{-1} \hat{\varepsilon}_{2 t} \operatorname{con} E\left(\varepsilon_{1 t} \mid \varepsilon_{2 t}\right)=\Omega_{12} \Omega_{22}^{-1} \varepsilon_{2 t}
$$

donde $\hat{\Omega}_{12}$ es la varianza promedio de largo plazo de dos lados entre las variables $\varepsilon_{1 t}$ y $\varepsilon_{2 t}, \Omega_{22}$ es la varianza de largo plazo de dos lados de $\varepsilon_{2 t}$ y finalmente $\varepsilon_{2 t}$ es la primera diferencia de los residuos obtenidos de (2). Por otro lado, $\lambda_{12}^{*}$ es el parámetro que corrige la estimación

$$
\hat{\Lambda}_{12}^{*}=\hat{\Lambda}_{12}-\hat{\Omega}_{12} \hat{\Omega}_{22}^{-1} \hat{\Lambda}_{22}
$$

donde $\Lambda_{12}$ es la varianza de largo plazo de un solo lado entre las variables $\varepsilon_{1 t}$ y $\varepsilon_{2 t}, \Lambda_{22}$ es la varianza de largo plazo de un solo lado de $\varepsilon_{2 t}$, y el resto de los parámetros se definen de acuerdo con la ecuación 5.

\section{Análisis estadístico de las variables de estudio}

Las series de tiempo Producto Interno Bruto y exportaciones de Canadá, México y Estados Unidos de Norteamérica fueron obtenidas de la OCDE, con periodicidad trimestral en el periodo 1993-2019; los países Canadá y Estados Unidos de Norteamérica, tienen base 2012, y México 2013.

En la gráfica 1 se presentan el Producto Interno Bruto y las Exportaciones de Canadá, México y Estados Unidos de Norteamérica con periodicidad trimestral denominados en su moneda corriente. En el lado izquierdo se presenta las variables de estudio en forma de niveles y del otro lado con la primera diferencia. Se puede observar que los tres países tienen movimientos similares, aunque con sus excepciones, por ejemplo, en la crisis mexicana de 1995 no se tiene ningún efecto sobre los otros dos países; sin embargo, cuando en Estados Unidos de Norteamérica crecieron sus Exportaciones a principios de este siglo se observa que esto tuvo un mayor impacto sobre Canadá que en México y, finalmente, la crisis del 2008 iniciada en EU tuvo efectos en la mayor parte de los países del mundo, aunque en este caso se 
observa que tiene mayor impacto sobre las economías pequeñas como la mexicana, ya que se nota una mayor caída en las Exportaciones y el Producto interno bruto, no así en la economía canadiense donde sólo se advierte una pronunciada caída en las Exportaciones. En el lado derecho de las gráfica 1 , se introdujo la primera diferencia de las variables de estudio en donde claramente se aprecian dos cosas, México tiene una alta dependencia con respecto a sus Exportaciones dado que se mueven de forma coordinada, con excepción en la crisis del 1995, donde tiene movimiento contrarios y, segundo, en los países desarrollados como Canadá y Estados Unidos de Norteamérica su crecimiento económico no depende de sus Exportaciones, dado que no se mueven de forma similar como se puede observar en esta gráfica 1.

\section{Grafica 1}

Producto Interno Bruto y Exportaciones de los países Canadá (CA) México (MX) y Estados Unidos de Norteamérica (US)
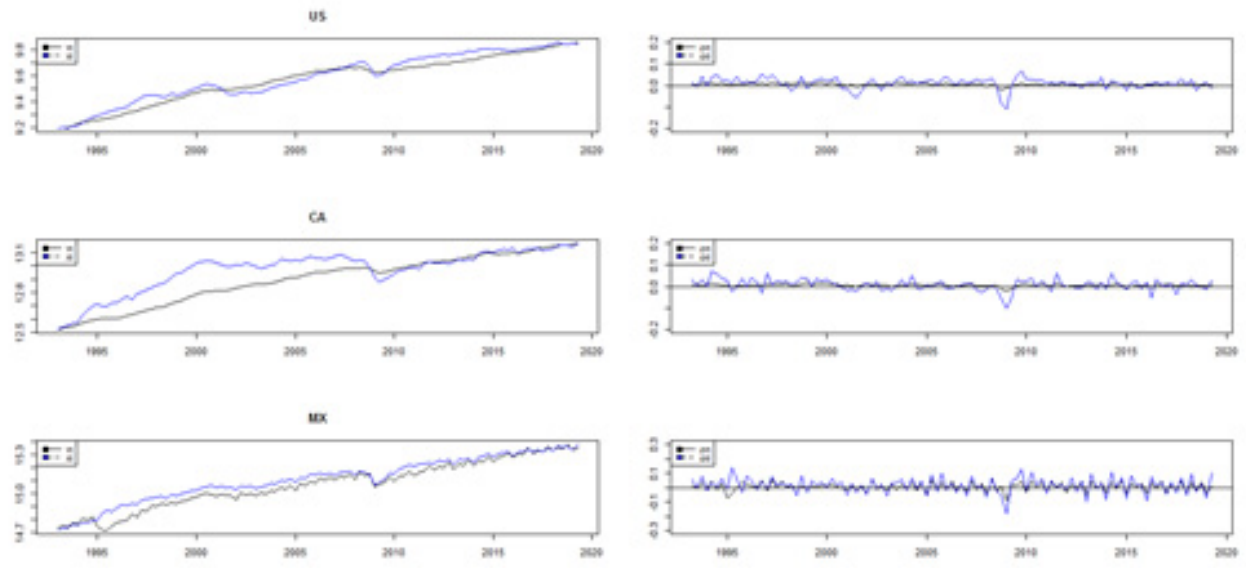

Fuente: elaboración propia con datos de la OECD 
En el cuadro 1 se presentan los estadísticos básicos de las variables Exportaciones y Producto Interno Bruto de los tres países, se puede observar que las Exportaciones representan una parte importante de las economías canadiense y mexicana, ya que representan el $27.2 \%$ y $25.8 \%$, respectivamente, para el periodo de 1993-2019; sin embargo, para la economía estadounidense sólo representa el 8.1\%. Un hecho interesante es que los tres países tienen un coeficiente de variación en su Producto Interno Bruto similar que oscila entre $17.3 \%$ al $17.9 \%$, aunque en la variable Exportaciones sólo México y Estados Unidos de Norteamérica tiene el coeficiente de más de $30 \%$.

\section{Cuadro 1}

Estadísticos básicos de los países Canadá (CA), México (MX) y Estados Unidos de Norteamérica (US)

\begin{tabular}{|c|c|c|c|c|c|c|c|}
\hline País & Variable & Media & $\begin{array}{c}\text { Des. } \\
\text { Est }\end{array}$ & Sesgo & Curtosis & $\begin{array}{c}\text { Jarque- } \\
\text { Bera }\end{array}$ & \multirow{2}{*}{ Correlación } \\
\hline \multirow{2}{*}{ Canada } & $\mathrm{Y}_{\mathrm{t}}$ & 404062 & 71340 & -0.22 & 1.93 & $5.91^{* *}$ & \multirow{2}{*}{$0.89^{*}$} \\
\cline { 2 - 7 } & $\mathrm{X}_{\mathrm{t}}$ & 109956 & 19469 & -0.91 & 3.04 & 14.45 & \\
\hline \multirow{2}{*}{ México } & $\mathrm{Y}_{\mathrm{t}}$ & 3542713 & 632457 & 0.09 & 2.06 & $4.08^{*}$ & \multirow{2}{*}{$0.99^{*}$} \\
\cline { 2 - 7 } & $\mathrm{X}_{\mathrm{t}}$ & 914126 & 361196 & 0.28 & 2.11 & $4.91^{* *}$ & \\
\hline \multirow{2}{*}{$\begin{array}{c}\text { Estados } \\
\text { UNidos }\end{array}$} & $\mathrm{Y}_{\mathrm{t}}$ & 14542 & 2521 & -0.21 & 2.06 & $4.69^{* *}$ & \multirow{2}{*}{$0.97^{*}$} \\
\cline { 2 - 6 } & $\mathrm{X}_{\mathrm{t}}$ & 1182 & 372 & 0.08 & 1.75 & $6.97^{* *}$ & \\
\hline \hline
\end{tabular}

Fuente: elaboración propia con datos de la OECD, las unidades están en moneda corriente.

${ }^{*},{ }^{* *} \mathrm{y}{ }^{* * *}$ representan estadísticamente significativo al 1\%, 5\% y 10\%, respectivamente, Des. Est. Es la desviación estándar

Por otra parte, El coeficiente de sesgo es negativo para Canadá en sus dos variables muestra que tuvo caídas no muy comunes en su economía, de allí que presente colas pesadas en la forma de la distribución, en cambio para México es positiva y marginal para su crecimiento económico. Es también importante observar que la economía canadiense y la estadounidense tienen un sesgo negativo similar y contrario al mexicano. El estadístico de curtosis indica que todas las variables siguen una forma de distribución de tipo platicurtica, lo cual quiere decir que durante este periodo los datos no se concentraron alrededor de la media, sino que estuvo dispersa, con excepción de las Exportaciones canadienses la cual es de tipo mesocúrtica. 
Los resultados de la prueba Jarque-Bera de normalidad muestran que las variables siguen una distribución normal al 95\% de confianza y el Producto Interno Bruto mexicano sigue una distribución normal al 99\%, sin dejar de observa que en las Exportaciones canadienses se rechaza la hipótesis nula de normalidad. Por último se aplicó el coeficiente de correlación de Pearson a las variables de estudio el cual resulto positivo y significativo al $99 \%$ de confianza, lo que significa que a medida que aumentan las Exportaciones se esperaría que aumentara el Producto Interno Bruto de las tres economías, lo cual coincide para el caso de México con lo obtenido por Balassa (1978).

En el cuadro 2 se presentan los resultados obtenidos al aplicar las pruebas de raíces unitarias y de estacionariedad a las dos variables de estudio de los tres países, en niveles y con primeras diferencias, con tendencia y sin tendencia. En las pruebas de raíces unitarias de Dickey-Fuller aumentada (1981, ADF) y Phillips-Perron (1988, PP) no se puede rechazar la hipótesis nula de raíces unitarias para todas las variables en nivel con y sin tendencia, aunque cabe mencionar que la variable $y_{t}$ para México es estadísticamente significativa al 99\% cuando se incluye la tendencia. La prueba de estacionariedad Kwiatkowski-Phillips-Schmidt-Shin (1992, KPSS) rechaza la hipótesis nula de estacionariedad cuando se aplica a las variables de nivel, con excepción de la variable asociada a México. Por último, cuando se aplican las pruebas ADF y PP a la primera diferencia de las variables de estudio, se rechaza la hipótesis nula de raíz unitaria al 95\% de confianza, lo cual indica que las variables son estacionarias con la primera diferencia, lo mismo sucede cuando se aplica la prueba de estacionariedad KPSS, aunque aquí se acepta la hipótesis nula de estacionariedad al 95\% de confianza. Por lo tanto, las variables en términos de nivel son $I(1)$ y las primeras diferencia son $I(0)$, por lo tanto las variables tienen el mismo orden de integración, el primer requisito para el proceso de cointegración. 


\section{Cuadro 2}

Pruebas de raíces unitarias de los países Canadá (CA), México (MX) y Estados Unidos de Norteamérica (US)

\begin{tabular}{|c|c|c|c|c|c|c|c|}
\hline \multirow[b]{2}{*}{ País } & \multirow[b]{2}{*}{ Variables } & \multicolumn{2}{|c|}{ ADF } & \multicolumn{2}{|c|}{ PP } & \multicolumn{2}{|c|}{ KPSS } \\
\hline & & $\begin{array}{c}\text { Sin } \\
\text { tendencia }\end{array}$ & $\begin{array}{c}\text { Con } \\
\text { tendencia }\end{array}$ & $\begin{array}{c}\text { Sin } \\
\text { tendencia }\end{array}$ & $\begin{array}{c}\text { Con } \\
\text { tendencia }\end{array}$ & $\begin{array}{c}\text { Sin } \\
\text { tendencia }\end{array}$ & $\begin{array}{c}\text { Con } \\
\text { tendencia }\end{array}$ \\
\hline \multicolumn{2}{|r|}{ Nivel } & & & & & & \\
\hline \multirow{2}{*}{ CA } & $\overline{Y_{t}}$ & -0.6725 & -2.1324 & -0.8656 & -1.9736 & 2.1794 & $0.3563^{* * *}$ \\
\hline & $X_{t}$ & -2.2824 & -2.4424 & -2.1473 & -2.2284 & 1.5754 & $0.3094^{* *}$ \\
\hline \multirow{2}{*}{ MX } & $Y_{t}$ & -0.4712 & $-3.6241^{*}$ & -0.4666 & $-6.4679^{*}$ & 2.1491 & $0.1413^{*}$ \\
\hline & $X_{t}$ & -0.0544 & $-2.9276^{* *}$ & 0.1655 & $-4.2433^{*}$ & 2.1386 & $0.2805^{* *}$ \\
\hline \multirow{2}{*}{ US } & $Y_{t}$ & -0.5315 & -2.0939 & -0.5678 & -1.7169 & 2.1552 & $0.3173^{* *}$ \\
\hline & $X_{t}$ & -0.7736 & -2.8342 & -0.6708 & $-2.8712^{* * *}$ & 2.1718 & $0.1351^{*}$ \\
\hline \multicolumn{8}{|c|}{ Primera diferencia } \\
\hline \multirow{2}{*}{ CA } & $\Delta Y_{t}$ & $-4.0817^{*}$ & $-4.0684^{*}$ & $-6.0492^{*}$ & $-6.0303^{*}$ & $0.1121^{*}$ & $0.0603^{*}$ \\
\hline & $\Delta X_{t}$ & $-3.2975^{* *}$ & $-3.2785^{* *}$ & $-8.6172^{*}$ & $-8.6484^{*}$ & $0.2433^{*}$ & $0.1308^{*}$ \\
\hline \multirow{2}{*}{ MX } & $\Delta Y_{t}$ & $-4.6135^{*}$ & $-4.5960^{*}$ & $-23.5857^{*}$ & $-23.4946^{*}$ & $0.0276^{*}$ & $0.0257^{*}$ \\
\hline & $\Delta X_{t}$ & $-5.3089^{*}$ & $-5.3182^{*}$ & $-18.8239^{*}$ & $-18.8138^{*}$ & $0.0824^{*}$ & $0.0404^{*}$ \\
\hline \multirow{2}{*}{ US } & $\Delta Y_{t}$ & $-3.8227^{*}$ & $-3.7993^{* *}$ & $-7.3171^{*}$ & $-7.2962^{*}$ & $0.1365^{*}$ & $0.1178^{*}$ \\
\hline & $\Delta X_{t}$ & $-4.8813^{*}$ & $-4.8585^{*}$ & $-6.7989^{*}$ & $-6.7639 *$ & $0.0359^{*}$ & $0.0355^{*}$ \\
\hline
\end{tabular}

Fuente: elaboración propia con datos de la OECD.

${ }^{*}, * * \mathrm{y}{ }^{* *}$ representan estadísticamente significativo al 1\%,5\% y $10 \%$, respectivamente.

\section{Estimación del modelo por medio de mínimos cuadrados totalmente modificados}

En el apartado anterior se observó que las variables en estudio tenían una tendencia estocástica que resultaron ser de tipo I(1) de acuerdo a las pruebas aplicadas. Lo que sigue es saber si estas tienen una tendencia estocástica en común, para lo cual se aplicará el proceso de cointegración de Engle y Granger (1987), en donde primero se necesita realizar la estimación del modelo propuesto por las ecuaciones (5) y (6) del apartado II. Primero Engle y Granger (1987) mencionan que dos variables tienen orden de integración $I(1)$, si en forma individual son $I(1)$ y además la combinación lineal de las variables es $I(0)$. En el cuadro 3 se presentan los resultados de la estimación 
por método FMOLS ${ }^{4}$ para obtener los residuos e indagar si están cointegradas las variables.

En el cuadro 3 se presentan los resultados de la estimación por el método propuesto y como se puede observar los tres parámetros son estadísticamente significativos al 99\% de confianza, esto de acuerdo con la prueba de significancia individual. Además, como se puede observar el parámetro asociado a las Exportaciones mexicanas nos dice que por cada peso que aumentan éstas, el Producto Interno Bruto mexicano aumenta en dos pesos.

\section{Cuadro 3}

Resultado de la estimación por medio del método FMOLS.

\begin{tabular}{|c|c|c|c|c|}
\hline País & Parámetro & $\begin{array}{c}\text { Desviación } \\
\text { estandar }\end{array}$ & $\boldsymbol{t}$ & $\boldsymbol{P}$ \\
\hline CA & 3.3435 & 0.3627 & 9.2191 & 0.00 \\
\hline MX & 1.7485 & 0.0432 & 40.4362 & 0.00 \\
\hline US & 6.6223 & 0.3366 & 19.6735 & 0.00 \\
\hline
\end{tabular}

Fuente: elaboración propia con datos de la OECD.

Cabe mencionar que aunque las Exportaciones canadienses y estadounidenses, no siguen una trayectoria similar a su producto interno bruto, los resultados de la estimación muestran que en estos países el impacto por aumentar esta variable sobre el producto es muy superior a la observada en la variable mexicana. Es decir, si en los Estados Unidos de Norteamérica aumentara un dólar las Exportaciones, su Producto Interno Bruto aumentaría siete dólares, de forma similar se puede explicar el caso de Canadá. Lo siguiente es ver si los residuos son $I(0)$ y por ende estacionarios. De acuerdo con Marin (1992), si las dos variables están cointegradas, la relación dinámica entre las variables de estudio puede no ser especificada.

\footnotetext{
${ }^{4}$ El modelo estocástico con la metodología de estimación FMOLS fue el siguiente $y_{t}=\alpha_{0}+\alpha_{1} x_{t}+\mu_{t}$
} 
En el cuadro 4 se muestran los resultados de las pruebas de raíces unitarias ADF y PP a los residuos ${ }^{5}$ obtenidos de la estimación por medio del método FMOLS. Los residuos asociados a Canadá en el cuadro 4 muestra que no se puede rechazar la hipótesis nula en ambas pruebas, lo cual quiere decir que los residuos de la regresión tienen una raíz unitaria por lo tanto no es estacionaria, entonces las series de tiempo Producto Interno Bruto y las Exportaciones no están cointegradas. Por otro lado, cuando se realizaron las mismas pruebas a los residuos asociados al modelo econométrico mexicano, se rechazó la hipótesis nula al 95\% de confianza, lo cual supone que los residuos son ruido blanco y, por ende, son estacionarios, de ahí que las variables Producto Interno Bruto y Exportaciones de México están cointegradas al menos por este método. Por último, para los residuos del caso de los Estados Unidos de Norteamérica, se rechaza la hipótesis nula al 90\% de confianza, de ahí que por este método indica que las variables de este país están cointegradas.

\section{Cuadro 4}

Pruebas de raíces unitarias a los residuos de la regresión

\begin{tabular}{lll}
\hline \multicolumn{1}{c}{ País } & \multicolumn{1}{c}{ ADF } & PP \\
\hline \hline CA & -1.2498 & -1.4318 \\
MX & $-3.2010^{* *}$ & $-3.3837 * *$ \\
US & $-1.7120^{* * *}$ & $-1.6531 * * *$ \\
\hline
\end{tabular}

Fuente: elaboración propia con datos de la OECD *,** $\mathrm{y}^{* * *}$ representan estadísticamente significativo al $1 \%, 5 \%$ y $10 \%$, respectivamente,

En el cuadro 5 se presentan los estadísticos $t$ y $z$ de la prueba de cointegración propuesta por Engle y Granger (1987) y Phillips y Ouliaris (1990), ${ }^{6}$ ambas pruebas se basan en el análisis de los residuos, la diferencia se encuentra

\footnotetext{
${ }^{5}$ Engle y Granger (1987) mencionan que si $x_{t}$ y $y_{t}$ son ambas $I(d)$, entonces es generalmente cierto que la combinación lineal sea $z_{t}=x_{t}-a y_{t}$, donde $z_{t}$ es un ruido blanco. Por otro lado, las pruebas de cointegración propuestas por estos autores fueron la Durbin-Watson (DW), Dickey-Fuller (DF) y DickeyFuller Aumentada (ADF) para modelos uniecuacionales y vectores autoregresivos.

${ }^{6}$ Phillips y Ouliaris (1990) propusieron dos clases de pruebas de cointegración, una para modelo uniecuacionales y otra para vectores autoregresivos, por ejemplo $Z_{a^{\prime}} Z_{t^{\prime}}$ proporción de la varianza $\left(P_{u}\right)$ y la prueba de traza multivariante $\left(P_{z}\right)$.
} 
en el cálculo de la varianza de largo plazo, por esta razón a la primera se considera de tipo paramétrica y a la segunda de tipo no paramétrica. En este cuadro se puede observar que las variables pertenecientes a México, el Producto Interno Bruto y las Exportaciones, según estas pruebas no se puede aceptar la hipótesis nula de no cointegración; sin embargo, para los otros dos países se acepta dicha hipótesis. Por lo tanto se considera que las variables están cointegradas. En resumen, las variables Producto Interno Bruto y Exportaciones de México, tienen orden de integración I(1) de acuerdo con el cuadro 2. En segundo lugar, el signo de la regresión indica que estas variables tienen una relación positiva de acuerdo con el cuadro 3 y, tercero, se tiene una relación de largo plazo dado que estas variables están cointegradas por lo visto en los cuadros 4 y 5 .

\section{Cuadro 5}

Prueba de cointegración de Engle y Granger (1987) y Phillips y Ouliaris (1990)

\begin{tabular}{|c|c|c|c|c|c|}
\hline \multirow{2}{*}{ País } & \multirow{2}{*}{ Variables } & \multicolumn{2}{|c|}{ Engle- Granger } & \multicolumn{2}{|c|}{ Phillips-Ouliaris } \\
\hline & & $Z_{\alpha}$ & $Z_{t}$ & $Z_{\alpha}$ & $Z_{t}$ \\
\hline \multirow[t]{2}{*}{ CA } & $Y_{t}$ & -1.6947 & -7.0358 & -1.3946 & -3.6086 \\
\hline & $X_{t}$ & -2.3482 & -12.3197 & -2.2269 & -6.7322 \\
\hline \multirow[t]{2}{*}{ MX } & $Y_{t}$ & $-3.2782^{* *}$ & $-43.8467^{*}$ & $-3.5603^{* *}$ & $-25.1831^{* *}$ \\
\hline & $X_{t}$ & $-3.2311^{* *}$ & $-65.1160^{*}$ & $-3.4441^{* *}$ & $-24.3997^{* *}$ \\
\hline \multirow[t]{2}{*}{ US } & $Y_{t}$ & -1.5105 & -5.2547 & -1.6334 & -5.3437 \\
\hline & $X_{t}$ & -1.5736 & -5.5886 & -1.6899 & -5.6859 \\
\hline
\end{tabular}

Fuente: elaboración propia con datos de la OECD.

${ }^{*},{ }^{* *} \mathrm{y}{ }^{* * *}$ representan estadísticamente significativo al $1 \%, 5 \%$ y $10 \%$, respectivamente.

En el cuadro 6 se muestran los resultados de la prueba de causalidad de Granger, junto con el parámetro asociado al mecanismo de corrección de error ${ }^{7}(\mathrm{MCE})$. Los resultados muestran que en ambos casos se rechaza la hipótesis nula, de ahí que la relación entre el Producto Interno Bruto y las Exportaciones de México sea de tipo bidireccional, es decir, cuando los

\footnotetext{
${ }^{7}$ El modelo estocástico para saber si el MCE es significativo fue $\Delta y_{t}=\alpha_{0}+\alpha_{1} \Delta x_{t}+\alpha_{2} \mu_{t-1}+\varepsilon_{t}$.
} 
encargados de diseñar e instrumentar la política económica en México estimulan a las Exportaciones, también están promoviendo un aumento en el Producto Interno Bruto y viceversa, aunado a estas evidencias se tiene que las variables se afectan en el corto y largo plazo de acuerdo con los resultados de los cuadro 3 y 6, el último muestra que el mecanismo de corrección del corto plazo es estadísticamente significado al 99\%.

Cuadro 6

Prueba de causalidad de Granger

\begin{tabular}{cccc}
\hline & $\boldsymbol{Y}_{\boldsymbol{t}}$ no causa Granger $\boldsymbol{X}_{\boldsymbol{t}}$ & $\boldsymbol{X}_{\boldsymbol{t}}$ no causa Granger $\boldsymbol{Y}_{\boldsymbol{t}}$ & MCE \\
\hline \hline$C ; \Delta z_{t}$ & 14.3639 & 4.1469 & 0.2803 \\
& $(0.0000)$ & $(0.0187)$ & $(0.0001)$ \\
\hline \hline
\end{tabular}

Fuente: elaboración propia con datos de la OECD, entre paréntesis la probabilidad.

\section{Conclusiones}

En este documento se realizó un estudio de la afectación de las Exportaciones al Producto Interno Bruto de Canadá, México y los Estados Unidos de Norteamérica, para el periodo 1993-2019, con datos trimestrales, con moneda corriente de los países. Primero se realizó un análisis gráfico en donde se mostró que en la economía mexicana, las Exportaciones siguen el mismo sendero que su Producto interno bruto, con excepción de la crisis de 1995, donde se registraron movimientos contrarios. En las otras dos economías no se observaron los mismos movimientos. Aunado a esto, en el cuadro 2 se mostró la alta dependencia de la economía mexicana con respecto a las Exportaciones con alrededor del $40 \%$ para este periodo.

Posteriormente se encontró que las variables eran de orden de integración I(1), en los tres casos, aunque el Producto Interno Bruto de México era estacionario cuando la pruebas de raíces unitaria y de estacionariedad se aplicaban con tendencia al 90\% de confianza. Luego, se encontró que aunque la economía mexicana tiene alta dependencia con respecto a las Exportaciones, éstas ejercen una influencia menor sobre la actividad económica ya que por cada peso que aumentan las Exportaciones el Producto Interno Bruto sólo aumenta en 1.75 pesos mexicanos, esto a diferencia de resto de los países 
que son de 3.35 y 6.62 dólares (canadienses y americanos), respectivamente. Después, cuando se aplicó las pruebas de cointegración se encontró que sólo las variables de estudio de la economía mexicana estaban cointegradas, es decir, tenían una relación en el largo plazo. Por último se efectuaron las pruebas de causalidad de Engle-Granger (1987) y Phillips-Ouliaris (1990) a las variables que estaban cointegradas de orden $I(1)$ y se encontró una relación bidireccional entre las Exportaciones y Producto interno bruto, de ahí que las políticas económicas que busquen incrementar a la variable Exportaciones estarán también buscando el crecimiento económico en el corto y largo plazo en virtud de los resultados de los cuadros 3 y 6 .

\section{Bibliografía}

Andrews, D. W. (1991). Heteroskedasticity and autocorrelation consistent covariance matrix estimation. Econometrica, 59(3): 817-858.

Andrews, D. W. y J. C. Monahan (1992). An improved heteroskedasticity and autocorrelation consistent covariance matrix estimator. Econometrica, 60(4): 953-966.

Awokuse, T. O. (2003). Is the export-led growth hypothesis valid for Canada? Canadian Journal of Economics, 36(1): 126-136.

Awokuse, T. O. (2008). Trade openness and economic growth: is growth export-led or import-led? Applied Economics, 40(2): 161-173.

Balassa, B. (1978). Exports and economic growth: further evidence. Journal of Development Economics, 5(2): 181-189.

Borensztein, E.; J. De Gregorio y J. W. Lee (1998). How does foreign direct investment affect economic growth? Journal of International Economics, 45(1): 115-135. 
Dhawan, U. and B. Biswal (1999). Re-examining export-led growth hypothesis: a multivariate cointegration analysis for India. Applied Economics, 31(4): 525-530.

Dickey, D. A. and W. A. Fuller (1981). Likelihood ratio statistics for autoregressive time series with a unitroot. Econometrica, 49(4): 1057-1072.

Engle, R. F. and C. W. Granger (1987). Co-integration and error correction: representation, estimation, and testing. Econometrica, 55(2): 251-276.

Johansen, S. (1988). Statistical analysis of cointegration vectors. Journal of Economic Dynamics and Control, 12(2-3): 231-254.

Johansen, S. (1991). Estimation and hypothesis testing of cointegration vectors in Gaussian vector autoregressive models. Econometrica, 59 (6) 1551-1580.

Kwan, A. C. and J. A. Cotsomitis (1991). Economic growth and the expanding export sector: China 1952-1985. International Economic Journal, 5(1): 105-116.

Kwiatkowski, D.; Phillips, P. C.; Schmidt, P. and Y. Shin (1992). Testing the null hypothesis of stationarity against the alternative of a unit root: How sure are we that economic time series have a unit root? Journal of Econometrics, 54(13): 159-178.

Lee, C. C. and P. C. Phillips (1994). An ARMA prewhitened long-run variance estimator. Manuscript, Yale University.

Marin, D. (1992). Is the export-led growth hypothesis valid for industrialized countries? Review of Economics and Statistics, 74(4): 678-688.

Michaely, M. (1977). Exports and growth: an empirical investigation. Journal of Development Economics, 4(1): 49-53.

Newey, W. K. and K. D. West (1987). A simple, positive semi-definite, heteroskedasticity and autocorrelation consistent covariance matrix. Econometrica, 55(3): 703-708.

Panas, E. and G. Vamvoukas (2002). Further evidence on the export-led growth hypothesis. Applied Economics Letters, 9(11): 731-735.

Phillips, P. C. and B. E. Hansen (1990). Statistical inference in instrumental variables regression with I(1) processes. Review of Economic Studies, 57(1): 99-125.

Phillips, P. C. and S. N. Durlauf (1986). Multiple time series regression with integrated processes. Review of Economic Studies, 53(4): 473-495.

Phillips, P. C. and P. Perron (1988). Testing for a unit root in time series regression. Biometrika, 75(2): 335-346.

Phillips, P. C. and S. Ouliaris (1990). Asymptotic properties of residual based tests for cointegration. Econometrica, 58(1): 165-193.

Rodríguez-Benavides, D. and F. Venegas-Martínez (2011). Efectos de las exportaciones en el crecimiento económico de México: un análisis de cointegración, 1929-2009. EconoQuantum, Revista de Economía y Negocios, 7(2): 55-71. 
Shan, J. and F. Sun (1998, a). Export-led growth hypothesis for Australia: an empirical re-investigation. Applied Economics Letters, 5(7): 423-428.

Shan, J. and F. Sun $(1998, b)$. On the export-led growth hypothesis: the econometric evidence from China. Applied Economics, 30(8): 1055-1065.

Shan, J. and K. Wilson (2001). Causality between trade and tourism: empirical evidence from China. Applied Economics Letters, 8(4): 279-283.

Shirazi, N. S. and T. A. A. Manap (2005). Export-led growth hypothesis: Further econometric evidence from South Asia. The Developing Economies, 43(4): 472-488.

Stock, J. H. and M. W. Watson (1993). A simple estimator of cointegrating vectors in higher order integrated systems. Econometrica, 61(4): 783-820.

Tyler, W. G. (1981). Growth and export expansion in developing countries: Some empirical evidence. Journal of Development Economics, 9(1): 121-130.

Wang, Q., and N. Wu (2012). Long-run covariance and its applications in cointegration regression. Stata Journal, 12(3): 515.

World Bank. 1987. World Development Report 1987: publication summary. Informe sobre el desarrollo mundial 1987. Resumen de una publicacion (Spanish). World Development Report, World Development Indicators. Washington, DC, World Bank Group. 